\title{
Cloning and Expression of Anthocyanin Biosynthesis Related Gene RrMYB6 in Rosa rugosa
}

\author{
Kai Zou", Yang Wang\#, Mingyuan Zhao, Lanyong Zhao*, Zongda Xu* \\ Forestry College of Shandong Agricultural University, Tai'an, China \\ Email: *sdzly369@163.com, *xuzoda@163.com
}

How to cite this paper: Zou, K., Wang, Y., Zhao, M.Y., Zhao, L.Y. and Xu, Z.D. (2018) Cloning and Expression of Anthocyanin Biosynthesis Related Gene RrMYB6 in Rosa rugosa. Agricultural Sciences, 9, 374-383. https://doi.org/10.4236/as.2018.93026

Received: January 30, 2018

Accepted: March 24, 2018

Published: March 27, 2018

Copyright $\odot 2018$ by authors and Scientific Research Publishing Inc. This work is licensed under the Creative Commons Attribution International License (CC BY 4.0).

http://creativecommons.org/licenses/by/4.0/

\section{(c) (i) Open Access}

\begin{abstract}
$\mathrm{R}_{2} \mathrm{R}_{3}$-MYB transcription factor plays an important role in plant anthocyanin synthesis. Based on the transcriptional database of Rosa rugosa, one MYB transcription factor related to floral color, $\operatorname{RrMYB6}$, was cloned. By using bioinformatics analysis method, cloning MYB gene and analyzing its function in anthocyanin biosynthesis regulation, we hope to lay a solid foundation for new color variety breeding of $R$. rugosa. Using the $R$. rugosa "Zi zhi" as the material, we obtained the total length of cDNA of RrMYB6 by RT-PCR and RACE. By analyzing its bioinformatics, we found that the formula of the protein was $\mathrm{C}_{1491} \mathrm{H}_{2368} \mathrm{~N}_{452} \mathrm{O}_{470} \mathrm{~S}_{17}$, molecular weight was $34690.97 \mathrm{Da}$, the theoretical pI was 8.74. In addition, it belonged to unstable protein with an unstable index at 50.59, and it was also a hydrophilic protein with the total average hydrophobic index at -0.847 . In the secondary structure of $R r M Y B 6$ protein, the Alpha helix accounted for $32.35 \%$, random coil was $47.39 \%$, extended strand was $11.11 \%$, and beta turn was $9.15 \%$. The sequence analysis showed that RrMYB6 had a typical $\mathrm{R}_{2} \mathrm{R}_{3}$-MYB domain and bHLH binding domain, and it also had an N1, C1, C2 inhibitory motif, belonging to the Sg4 subfamily MYB protein. What's more, evolutionary analysis indicated that the $\operatorname{RrMYB6}$ protein was closely related with the MYB protein in Rosacea family, while it was far from those in other families. The expression analysis showed that RrMYB6 protein decreased with the color of petals deeping, and its expression was the lowest in the petals while the highest in stamens. According to the above results, it was speculated that RrMYB6 was involved in regulating the anthocyanin synthesis of $R$. rugosa, which belonged to negative regulatory mechanism.
\end{abstract}

\section{Keywords}

Rosa rugosa, Anthocyanin, R2R3-MYB, Gene Expression

\#These authors contribute equally. 


\section{Introduction}

Rosa rugosa, as a deciduous shrub in the Rosacea family, has high ornamental value and fragrant aroma, and plays an important role in landscape architecture. But the color of $R$. rugosa is simple, most is red, pink and white, and is fresh in other color, which have seriously restricted its application in gardens for a long time. In higher plants, the color of petals, tissues and fruits is determined by anthocyanin, which is a secondary metabolite belonging to flavonoids. Anthocyanins are synthesized through three steps with a series of enzymes [1]. The anthocyanins pathway has been explored in depth in Petunia hybrid [2], Zea mays [3], Antirrhinum majus [4] and so on. In higher plants, most anthocyanin biosynthesis is regulated by the combination of many transcription factors in different time and space. TFs like $\mathrm{R}_{2} \mathrm{R}_{3}-\mathrm{MYB}, \mathrm{bHLH}, \mathrm{WD}-40$ can regulate anthocyanin biosynthesis by forming a MBW ternary complex [5] [6]. As a key transcription factor, the up-regulation of $\mathrm{R}_{2} \mathrm{R}_{3}$-MYB can activate the expression of one or more structural genes involved in anthocyanin synthesis, so leads to anthocyanin accumulating. The related genes have been cloned and analyzed in its function from species such as Epimedium sagittatum [7], Pyrus pyrifolia [8] and Prunus avium L. [9]. At present, there are few reports on the anthocyanin biosynthesis mechanism of $R$. rugose, so we don't know exactly how it works. Based on the rose transcriptome data, we cloned one $\mathrm{R}_{2} \mathrm{R}_{3}$-MYB gene related with anthocyanin synthesis and figured out its function by bioinformatics analysis and real-time PCR to provide foundation for the regulation mechanism of anthocyanin synthesis in the petals of $R$. rugosa.

\section{Materials and Methods}

The experiment was conducted from April 2016 to January 2017 at the flower germplasm resource nursery of Shandong Agricultural University and the Flower Institute of Forestry College.

\subsection{Plant Materials}

The plant material, Chinese representative $R$. rugosa "Zi zhi”, “Bai zizhi”, "Fen zizhi" were from the rose germplasm resources garden at Shandong Agricultural College. R. rugosa "Zi zhi”, "Bai zizhi”, "Fen zizhi” are the most representative traditional rose in China. And the colors of three are purple, white and pink, respectively. From April to May, 2016, the petals in initial opening stage of a single plant with strong growth potential and stable hues in above three cultivars were collected for the experiment of gene cloning and difference expression; Sepals, stems, leaves, stamens, pistils and petals samples for gene spatial expression analysis were collected from $R$. rugosa "Zi zhi" at full opening stage. All samples were collected directly frozen with liquid nitrogen, and finally stored at $-80^{\circ} \mathrm{C}$ until used. 


\subsection{Methods}

\subsubsection{RNA Extraction and Gene Cloning}

The extraction of RNA from various tissues of plants was operated according to the instructions of EASYspin plant RNA rapid extraction kit, and its concentration and purity were determined by ultraviolet spectrophotometer. At the same time, the integrity of RNA was detected by $1 \%$ agarose gel electrophoresis. cDNA was synthesized by RNA reverse transcription kit according to the description of abm reverse transcription kit. Fragment amplification: according to the data of rose transcriptome, the relevant MYB gene fragments were selected, and specific primers were designed by Oligo 7.0 software (Table 1). PCR amplification was carried out with the synthesized cDNA as the template. The reaction system of PCR was as follows: Mix $12.5 \mathrm{ul}$, the target gene upstream and downstream primers each $1 \mathrm{ul}$, template cDNA $1 \mathrm{ul}$, sterilization $\mathrm{ddH}_{2} \mathrm{O}$ supplement to $25 \mathrm{ul}$; The PCR reaction conditions are as follows: $94^{\circ} \mathrm{C}$ for $5 \mathrm{~min} ; 94^{\circ} \mathrm{C}$ for 30 s, $53^{\circ} \mathrm{C}$ for $30 \mathrm{~s}$, and $72^{\circ} \mathrm{C}$ for $1 \mathrm{~min}$ for a total of 35 cycles; and then extension at $72^{\circ} \mathrm{C}$ for $10 \mathrm{~min}$. Next, $1 \%$ agarose gel electrophoresis was used to detect the PCR products. The full-length amplification was carried out at $72^{\circ} \mathrm{C}$ for $10 \mathrm{~min}$. Specific primers (Table 1 ) were used to amplify the three races and the complete open reading frame (ORF). The reaction system and PCR reaction conditions were described above. The PCR product was reclaimed according to the description of Hipure Gel Pure DNA Mini Kit(Magen), then ligated with the carrier pMD18-T to transform Escherichia coli DH5 $\alpha$. Positive clones were selected and sequenced at BGI.

\subsubsection{Bioinformatics Analysis of RrMYB6}

BLASTX (NCBI) was used to study the homology of the nucleotide sequence and the deduced amino acid sequence. DNAMAN5.2.2 was used to conduct multiple sequence alignment. The ORF finder (NCBI) was used to search for an

Table 1. Primers used to clone and expression analysis of RrMYB6 in R. rugosa.

\begin{tabular}{cccc}
\hline Name & Primer sequence (5' to 3') & Purpose & $\begin{array}{c}\text { Annealing } \\
\text { temperatures } /{ }^{\circ} \mathrm{C}\end{array}$ \\
\hline 6-F & AAGCTCACACCAACAAAGGGG & Cloning of the & 63.6 \\
6-R & TCAAGGGTCTGTGAAATCTGT & Middle Fragment & 57.2 \\
6-3'-F & TGTCAGTGCACAAATGGCTTC & & 62.2 \\
B26 & GACTCGAGTCGACATCGATTTTTTTT & 3'RACE PCR & 70.4 \\
RrMYB6-F & ATGGGAAGGTCACCTTGCTGT & ORF PCR & 63.1 \\
RrMYB6-R & CTATGAATTCAAGGGTCTGTG & & 54.8 \\
Actin-F & CACTTAGCACCTTCCAGCAGATGT & & 64.0 \\
Actin-R & CTACAACAGCAGACCTGAGTTCACT & Real-time & 61.3 \\
RrMYB6-Q-F & CACCGAAGAAGAAGACGAG & Quantitative PCR & 56.1 \\
RrMYB6-Q-R & TTCATTGTCGGTTCGTCC & & 57.9 \\
\hline
\end{tabular}


open reading frame, and the Conserved Domains database (NCBI) was used to analyze the conserved domains. ExPaSy-SOPMA was used to predict protein secondary structure. The ProtParam Tool was used to analyze protein physical and chemical properties. Furthermore, the ProtScale was used to predict hydrophilic or hydrophobic protein proper-ties. The NetPhos 3.1 Server was used to predict potential protein phosphorylation sites, and the NetOGlyc 4.0 Server was used to predict potential protein glycosylation sites. In order to study the evolutionary relationship between $\operatorname{RrMYB6}$ and $M Y B 6$ proteins in other species, we chose 13 MYB6 genes in different spices with high level of homology to RrMYB6 by BLAST, and constructed evolutionary tree with them by MEGA5.0 software. The phylogenetic tree was constructed according to the neighbor-joining method, and tested by bootstrap, which was repeated 1000 times.

\subsubsection{Expression Analysis of RrMYB6 in Different Tissues and Different Cultivars}

Using CFX96TM Real-Time System RT-qPCR instrument and SYBR ${ }^{\circledR}$ Premix Ex TaqTM kit as template for real-time fluorescence quantitative analysis. The primers and internal reference primers (Actinin) are shown in Table 1. The reaction system is: Mix10ul, cDNA $1 \mathrm{ul}, 0.4 \mathrm{ul}$ each of upstream and downstream primer, adding sterilizing $\mathrm{ddH}_{2} \mathrm{O}$ up to $20 \mathrm{ul}$. The reaction procedure was: predenaturation at $95^{\circ} \mathrm{C}$ for $30 \mathrm{~s} ; 95^{\circ} \mathrm{C}$ for $30 \mathrm{~s}, 60^{\circ} \mathrm{C}$ for $30 \mathrm{~s}$ for a total of 39 cycles. The cycle threshold $(\mathrm{Ct})$ value for each PCR reaction was calculated. After completion of the amplification steps, the melting curve was determined for each analysis. Gene transcripts were quantified using the comparative $\mathrm{Ct}$ method, which compares the transcript level of the target gene with that of the reference gene. The gene was set up with three repeats and the experimental data were processed by $2^{-\triangle \Delta C T}$. And the data analysis was graphed through software Prism 5.

\section{Results and Analysis}

\subsection{Cloning and Sequence Analysis of RrMYB6}

One R2R3-MYB transcription factor, $R r M Y B 6$, was cloned from the petals of Rosa rugosa "Zi zhi”. Its Genbank accession Nos: MG745778. The RrMYB6 gene has a full length of $1107 \mathrm{bp}$, an open reading frames (ORFs) with length of 921 bp (Figure 1), and encoding 306 amino acids.

Multiple sequence alignment analysis showed that the amino acid sequence encoded by RrMYB6 had five conserved regions, including R2R3-MYB domain, $\mathrm{C} 1$ and $\mathrm{C} 2$ suppressor motifs domain, PCCEK (N1) motif and the domain interacting with bHLH protein. It is inferred that RrMYB6 is a typical R2R3-MYB transcription factor (Figure 2). The evolutionary tree results revealed that RrMYB6 exhibited the highest homology to FVMYB6 in Fragaria vesca. And six $M Y B 6$ proteins from other plants in Rosaceae family, such as $M d M Y B 6$, PmMYB6 and so on, were shown to be closely related with $\operatorname{RrMYB6}$, and 


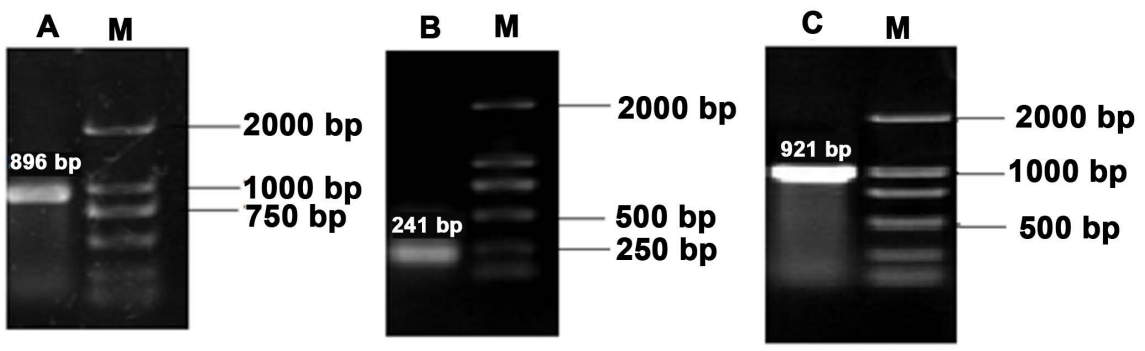

Figure 1. PCR amplification of RrMYB6. M:Marker; A: Intermediate ragment; B: 3'-RACE; C: Full-length fragment.

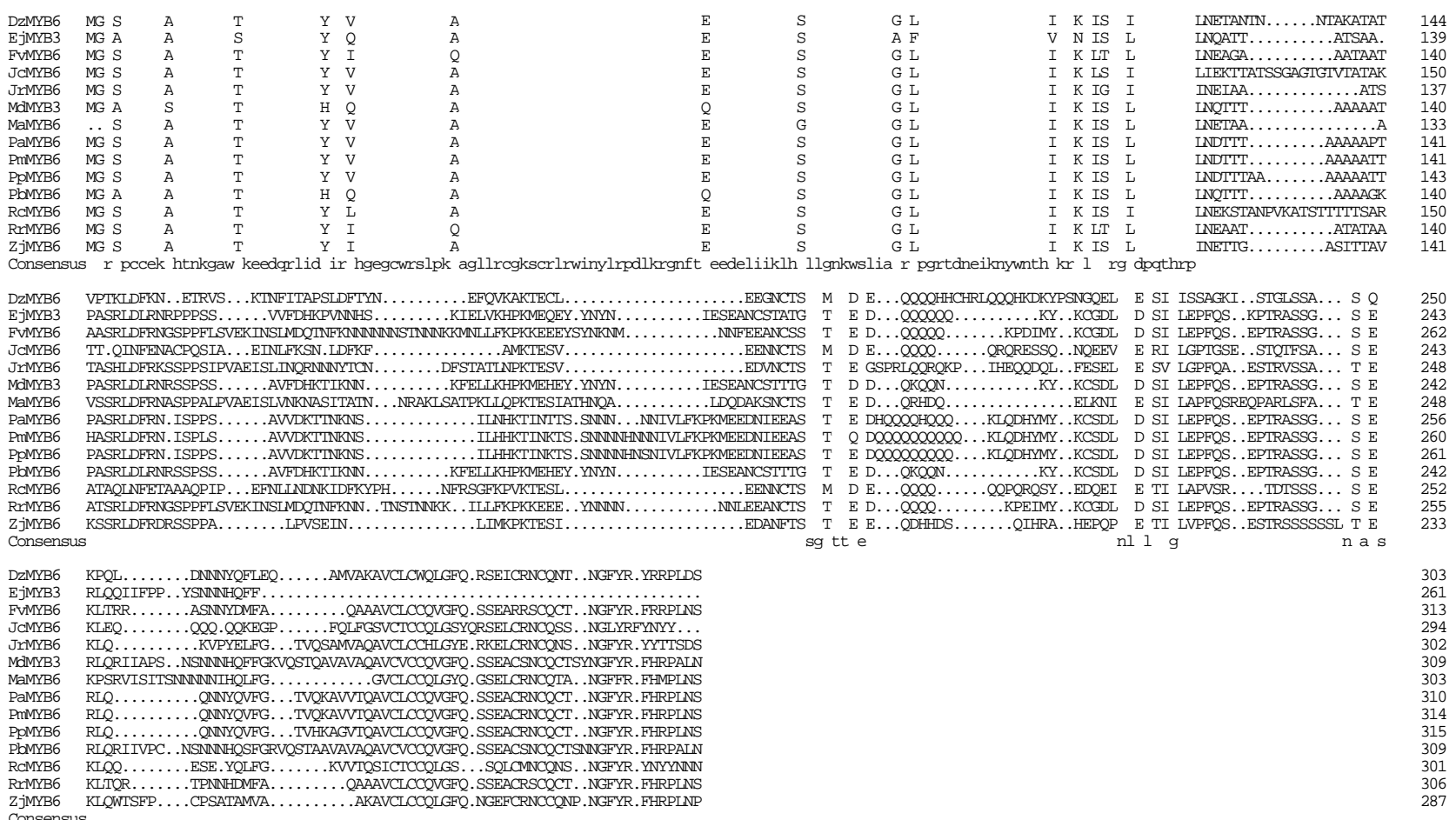

Figure 2. Multiple alignment of the RrMYB6 with other MYB TFs Notes: The red linear indicate the conserved R2-domain and R3-domain, the black linear indicate the conserved residuals interacting with bHLH proteins.

clustering in a clade. While RrMYB6 was relatively distant from MaMYB6, JrMYB6, and other $M Y B 6$ proteins in other families, which were clustered into another branch (Figure 3).

\subsection{Bioinformatics Analysis of RrMYB6 Gene}

The RrMYB6 gene encoded 306 amino acids. The protein had a molecular weight of $34690.97 \mathrm{Da}$, an isoelectric point of 8.74 , and its formula was $\mathrm{C}_{1491} \mathrm{H}_{2368} \mathrm{~N}_{452} \mathrm{O}_{470} \mathrm{~S}_{17}$. There were 42 basic amino acids (Arg + Lys), 35 acidic amino acids, and 229 neutral amino acids. It belonged to unstable protein with an unstable index at 50.59, and it was also a hydrophilic protein with the total average hydrophobic index at -0.847 . The secondary structure prediction results demonstrated that there was $99 \alpha$-helix, 145 random coil, 34 extended peptide 


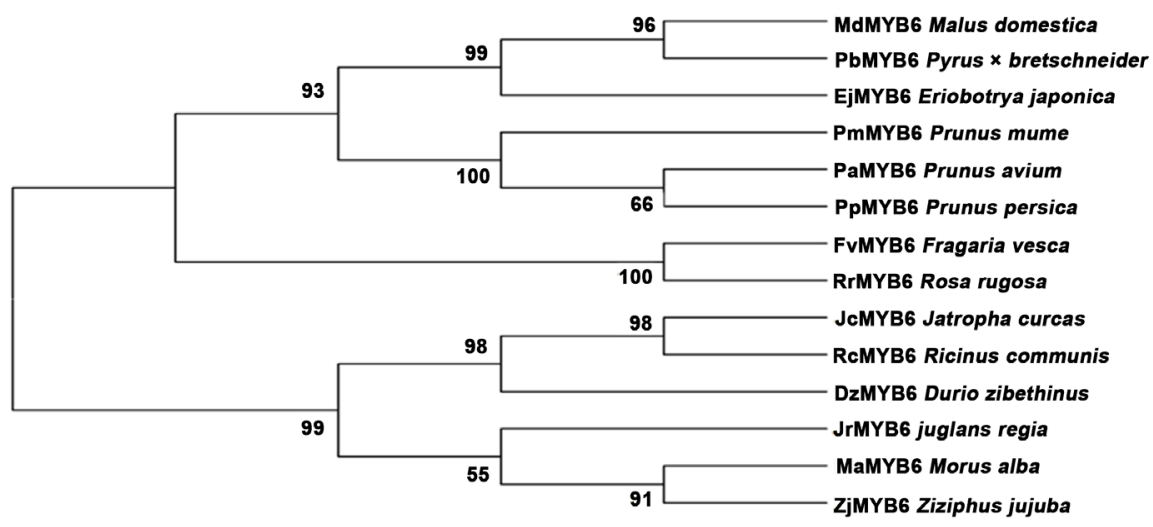

Figure 3. The phylogenetic tree derived from the alignment of amino acid sequences of RrMYB6 and other MYB6 TFs.

chain, and $28 \beta$-turn. The phosphorylation site prediction results demonstrated that there were 19 Ser phosphorylation sites, 22 Thr phosphorylation sites, and 6 Tyr phosphorylation sites, thus speculating that it was related to phosphorylation regulation.

\subsection{Expression Patterns of RrMYB6 in Different Tissues and Different Varieties}

Relative expression of $R r M Y B 6$ was profiled using q-PCR in $R$. rugose "Zi zhi" leaves, sepals, stems, stamens, pistils, and petals to analyze its tissue specificity, while it was also detected in the petals of $R$. rugose "Zi zhi", $R$. rugosa "Fen zizhi", $R$. rugosa "Bai zizhi" to analyze its variety specificity. The expression analysis of $R r M Y B 6$ in different tissues showed that $R r M Y B 6$ expressed differentially among stems, leaves, stamens, sepals, pistils and petals. RrMYB6 was more abundant in stamens than stems, leaves, petals, sepals and pistils. The highest expression level of RrMYB6 was observed in stamens, while it expressed slightly in sepals, pistils and petals, and almost didn't express in stems and leaves. In addition, the expression analysis of RrMYB6 in different varieties showed that the RrMYB6 decreased with the color deepening among the three cultivars, lowest in the petals of $R$. rugose "Zi zhi", followed by R. rugosa "Fen zizhi", and highest in R. rugosa "Bai zizhi" (Figure 4).

\section{Discussion}

One MYB transcription factor, RrMYB6, was cloned, and the sequence analysis of its protein showed that it contained a R2R3 domain, $\mathrm{C} 1$ and $\mathrm{C} 2$ suppressor motifs domain, PCCEK (N1) motif and the domain interacting with bHLH protein. Furthermore, its amino acid sequence is highly conserved at N1 motif and R2R3 domains, thus belonging to R2R3-MYB protein in Sg4 subfamily [10] [11] [12] [13] [14] [15]. Studies show that the C1 repression motif of Sg4 subfamily affect the secondary metabolism of plants by inhibiting the expression of cinnamic acid 4-hydroxylase gene [16] [17]. The R2R3-MYB proteins in different 


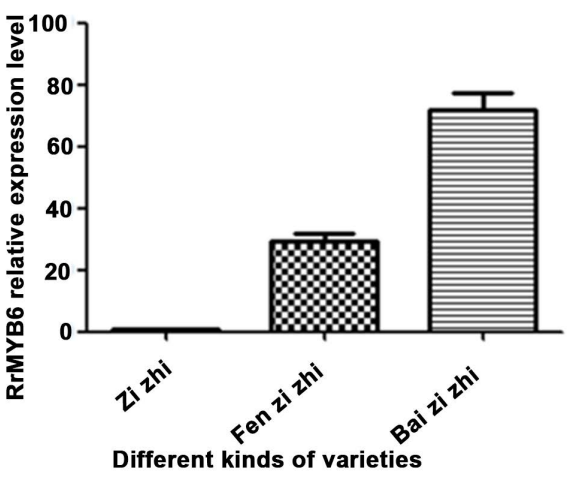

(a)

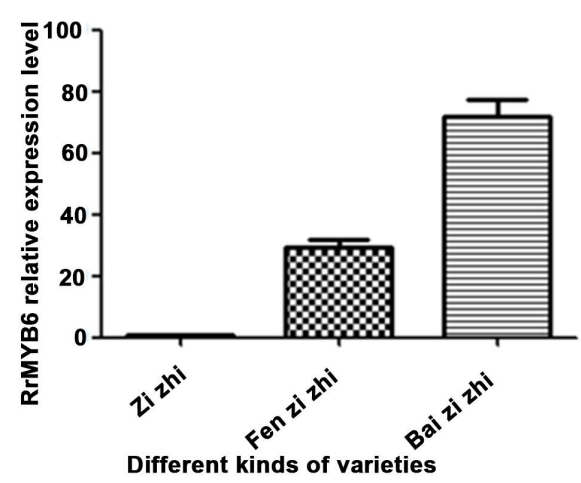

(b)

Figure 4. Relative expression levels of RrMYB6.

spices have different effects on anthocyanin synthesis. For example, the overexpression of FaMYB1 in tobacco made its flower color shallower with the anthocyanins decreased significantly [18], while the MdMYB3 isolated from Malus domstica exhibited oppositely [19] [20]. Evolutionary analysis showed that $R r M Y B 6$ shared the closet homology to $F_{V M Y B 6}$, so it was speculated that RrMYB6 could regulate the anthocyanin synthesis in $R$. rugosa. Specifically, whether it exhibited in a positive way or negative way needs further discussion.

The bioinformatics analysis showed that in the secondary structure of RrMYB6 protein, $\alpha$-helix and random coil both accounted for considerable proportion. However, the proportion of extended strand and $\beta$-turn was smaller. Studies have proved that $\alpha$-helix plays an important role in the $\mathrm{R}$ motif of the MYB domain. Each $\mathrm{R}$ motif is generally composed of three $\alpha$-helix, in which the $\alpha$-helix at the secondary and third position will form the HTH structure. And then combine with the first R motif, further forming a HTH domain with a hydrophobic core. What's more, the third alpha helices in $\mathrm{R}$ motif have a role of identifying DNA, which makes the MYB protein more specific. Therefore, it can be further determined that RrMYB6 gene belongs to R2R3-MYB type [21] [22]. The irregular curl structure is very beneficial to the binding of cell and water molecule, and both belong to hydrophilic protein. It can be inferred that both of them may protect the plant from osmotic stress. In addition, $R r M Y B 6$ contains a certain phosphorylation site, which may be related to the regulation of phosphorylation.

The qRT-PCR results showed that $R r M Y B 6$ expressed slightly in the petals of $R$. rugose "Zi zhi", followed by $R$. rugosa "Fen zizhi", and highly expressed in $R$. rugosa "Bai zizhi". Furthermore, $\operatorname{RrMYB6}$ exhibited a characteristic low expression in petals while expressed at a high level in stamen in the different tissues of R. rugosa "Zi zhi". Studies show that there are positive and negative mechanisms for anthocyanin biosynthesis regulation by MYB protein in plants [22]. The $M d M Y B$ isolated from Malus domestica regulates the synthesis of anthocyanin. Moreover, lightness is the essential factor in the regulation of biosynthetic gene by $M d M Y B$. Overexpression of $M d M Y B 10$ isolated from leaves and pulp can in- 
crease anthocyanin accumulation in apple seedlings. What's more, the overexpression of $M d M Y B 16, M d M Y B 17$ and $M d M Y B 111$ in tobacco could inhibit the activity of DFR promoter and thus inhibiting anthocyanin synthesis [23] [24]. Li also find that MYB protein has positive and negative mechanisms for anthocyanin synthesis in Morus alba [25]. The expression of RrMYB6 gene decreased with the deepening of petal color. And in the expression analysis of $R$. rugosa " $\mathrm{Zi}$ zhi" tissues, the expression of petals was very low, which was consistent with the analysis of expression among varieties. The gene expressed slightly in the petals, which may caused by different flowering stages. These results suggested that the regulation of RrMYB6 gene on anthocyanin biosynthesis in $R$. rugosa was a negative regulation mechanism. The regulation of genes involved in this mechanism needs further verification.

In conclusion, one R2R3-MYB TF, RrMYB6, was isolated from $R$. rugosa and was found to be involved in regulating anthocyanin biosynthetic pathway. The results of this study provided important information on the anthocyanin synthesis of $R$. rugosa. In future work, we will test whether the overexpression of RrMYB6 leads to anthocyanin accumulation in Arabidopsis thaliana and Nicotiana tabacum.

\section{Acknowledgements}

This work was funded by Shandong Province Agricultural Engineering project of breeding ([2014] No. 96).

\section{References}

[1] Buer, C.S., Imin, N. and Djordjevic, M.A. (2010) Flavonoids: New Roles for Old Molecules. Journal of Integrative Plant Biology, 52, 98-111. https://doi.org/10.1111/j.1744-7909.2010.00905.x

[2] Schwinn, K.E., Boase, M.R., Bradley, J.M., et al. (2014) MYB and bHLH transcription Factor Transgenes Increase Anthocyanin Pigmentation in Petunia and Lisianthus Plants, and the Petunia Phenotypes Are Strongly Enhanced under Field Conditions. Frontiers in Plant Science, 5, 603. https://doi.org/10.3389/fpls.2014.00603

[3] Ibraheem, F., Gaffoor, I., Tan, Q., et al. (2015) A Sorghum MYB Transcription Factor Induces 3-deoxyanthocyanidins and Enhances Resistance against Leaf Blights in Maize. Molecules, 20, 2388-2404. https://doi.org/10.3390/molecules20022388

[4] Schwinn, K., Venail, J., Shang, Y., et al. (2006) A Small Family of MYB-Regulatory Genes Controls Floral Pigmentation Intensity and Patterning in the Genus Antirrhinum. The Plant Cell, 18, 831-851. https://doi.org/10.1105/tpc.105.039255

[5] Ravaglia, D., Espley, R., Henry, K.R., et al. (2013) Transcriptional Regulation of Flavonoid Biosynthesis in Nectarine (Prunus persica) by a Set of $\mathrm{R}_{2} \mathrm{R}_{3}-\mathrm{MYB}$ Transcription Factors. BMC Plant Biology, 13, 68. https://doi.org/10.1186/1471-2229-13-68

[6] Li, L., Ban, Z.J., Li, X.H., et al. (2012) Differential Expression of Anthocyanin Biosynthetic Genes and Transcription Factor PcMYB10 in Pears (Pyrus communis L.). Plos One, 7, e46070. https://doi.org/10.1371/journal.pone.0046070

[7] Huang, W., Sun, W., Lv, H., et al. (2013) $\mathrm{A} \mathrm{R}_{2} \mathrm{R}_{3}$-MYB Transcription Factor from 
Epimedium sagittatum Regulates the Flavonoid Biosynthetic Pathway. PLoS ONE, 8, e70778. https://doi.org/10.1371/journal.pone.0070778

[8] Feng, S., Wang, Y., Yang, S., et al. (2010) Anthocyanin Biosynthesis in Pears Is Regulated by a $\mathrm{R}_{2} \mathrm{R}_{3}$-MYB Transcription Factor PyMYB10. Planta, 232, 245-255. https://doi.org/10.1007/s00425-010-1170-5

[9] Starkevic, P., Paukstyte, J., Kazanaviciute, V., et al. (2015) Expression and Anthocyanin Biosynthesis-Modulating Potential of Sweet Cherry (Prunus avium L.) MYB10 and bHLH Genes. PLOS ONE, 10, e0126991. https://doi.org/10.1371/journal.pone.0126991

[10] Zhao, J., Liu, R., Yang, F., et al. (2015) Cloning and Expression Analyses of R2R3-MYB Genes Related to Anthocyanin Biosynthesis in Rose. Scientia Agricultura Sinica, 48, 1392-1404.

[11] Xu, Z.S., Feng, K., Que, F., et al. (2017) A MYB Transcription Factor, DcMYB6, Is Involved in Regulating Anthocyanin Biosynthesis in Purple Carrot Taproots. Scientific Reports 7, Article ID: 45324. https://doi.org/10.1038/srep45324

[12] Butelli, E., Licciardello, C., Zhang, Y., Liu, J., et al. (2012) Retrotransposons Control Fruit-Specific, Cold-Dependent Accumulation of Anthocyanins in Blood Oranges. The Plant Cell, 24, 1242-1255. https://doi.org/10.1105/tpc.111.095232

[13] Yamagishi, M., Shimoyamada, Y., Nakatsuka, T., et al. (2010) Two $\mathrm{R}_{2} \mathrm{R}_{3}-\mathrm{MYB}$ Genes, Homologs of Petunia AN2, Regulate Anthocyanin Biosyntheses in Flower Tepals, Tepal Spots and Leaves of Asiatic Hybrid Lily. Plant and Cell Physiology, 51, 463-474. https://doi.org/10.1093/pcp/pcq011

[14] Xu, Z.S., et al. (2014) Transcript Profiling of Structural Genes Involved in Cyanidin-Based Anthocyanin Biosynthesis between Purple and Non-Purple Carrot (Daucus carota L.) Cultivars Reveals Distinct Patterns. BMC Plant Biology, 14, 1583-1588. https://doi.org/10.1186/s12870-014-0262-y

[15] Wang, H.Z., Qu, H.Y., Zhou, T.T., et al. (2017) Cloning and Expression Analysis of Anthocyanin Biosynthesis-Associated DFR and MYB Genes in Calyx of Eggplant (Solanum melongena L.). Scientia Agricultura Sinica, 50, 2781-2792.

[16] Jin, H., Cominelli, E., Bailey, P., et al. (2000) Transcriptional Repression by AtMYB4 Controls Production of UV-Protecting Sunscreens in Arabidopsis. The EMBO Journal, 19, 6150-6161. https://doi.org/10.1093/emboj/19.22.6150

[17] Lin, W.K., Bolitho, K., Grafton, K., et al. (2010) An $\mathrm{R}_{2} \mathrm{R}_{3}-\mathrm{MYB}$ Transcription Factor Associated with Regulation of the Anthocyanin Biosynthetic Pathway in Rosaceae. BMC Plant Biology, 10, 50. https://doi.org/10.1186/1471-2229-10-50

[18] Vimolmangkang, S., Han, Y., Wei, G., et al. (2013) An Apple MYB Transcription Factor, MdMYB3, Is Involved in Regulation of Anthocyanin Biosynthesis and Flower Development. BMC Plant Biology, 13, 176. https://doi.org/10.1186/1471-2229-13-176

[19] Aharoni, A., De, V.C.H., Wein, M., et al. (2001) The Strawberry FaMYB1 Transcription Factor Suppresses Anthocyanin and Flavonol Accumulation in Transgenic Tobacco. The Plant Journal, 28, 319-332. https://doi.org/10.1046/j.1365-313X.2001.01154.x

[20] Wang, L., Han, W., Xie, C., et al. (2013) Comparing the Acceptor Promiscuity of a Rosa hybrida Glucosyltransferase RhGT1 and an Engineered Microbial Glucosyltransferase OleD (PSA) toward a Small Flavonoid Library. Carbohydrate Research, 368, 73-77. https://doi.org/10.1016/j.carres.2012.12.012

[21] Liu, X.F., Li, F., Yin, X.R., et al. (2013) Recent Advances in the Transcriptional Reg- 
ulation of Anthocyanin Biosynthesis. Acta Horticulturae Sinica, 40, 2295-2306. https://doi.org/10.1007/s10114-013-2167-3

[22] Bendahmane, M., Dubois, A., Raymond, O., et al. (2013) Genetics and Genomics of Flower Initiation and Development in Roses. Journal of Experimental Botany, 64, 847-857. https://doi.org/10.1093/jxb/ers387

[23] Albert, N.W., Davies, K.M., Lewis, D.H., et al. (2014) A Conserved Network of Transcriptional Activators and Repressors Regulates Anthocyanin Pigmentation in Eudicots. The Plant Cell, 26, 962-980. https://doi.org/10.1105/tpc.113.122069

[24] Takos, A.M., Jaffe, F.W., Jacob, S.R., et al. (2006) Light-Induced Expression of a MYB Gene Regulates Anthocyanin Biosynthesis in Red Apples. Plant Physiology, 142, 1216-1232. https://doi.org/10.1104/pp.106.088104

[25] Lin, W.K., Micheletti, D., Palmer, J., et al. (2011) High Temperature Reduces Apple Fruit Colour via Modulation of the Anthocyanin Regulatory Complex. Plant, Cell \& Environment, 34, 1176-1190. https://doi.org/10.1111/j.1365-3040.2011.02316.x 